\title{
Recherches sur la morphogénèse d'un village de Bresse : Romenay (Saône-et-Loire)
}

\section{Antoine Guicheteau et Anne-Lise Bugnon-Labaune}

\section{(2) OpenEdition \\ 1 Journals}

Édition électronique

URL : https://journals.openedition.org/cem/15704

DOI : $10.4000 /$ cem. 15704

ISSN : 1954-3093

\section{Éditeur}

Centre d'études médiévales Saint-Germain d'Auxerre

\section{Référence électronique}

Antoine Guicheteau et Anne-Lise Bugnon-Labaune, «Recherches sur la morphogénèse d'un village de Bresse : Romenay (Saône-et-Loire) », Bulletin du centre d'études médiévales d'Auxerre / BUCEMA [En ligne], 22.2 | 2018, mis en ligne le 23 janvier 2019, consulté le 22 septembre 2022. URL : http:// journals.openedition.org/cem/15704; DOI : https://doi.org/10.4000/cem.15704

\section{Ce document a été généré automatiquement le 22 septembre 2022.}

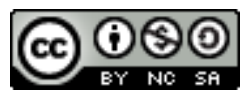

Creative Commons - Attribution - Pas d'Utilisation Commerciale - Partage dans les Mêmes Conditions 4.0 International - CC BY-NC-SA 4.0

https://creativecommons.org/licenses/by-nc-sa/4.0/ 


\title{
Recherches sur la morphogénèse d'un village de Bresse : Romenay (Saône-et-Loire)
}

\author{
Antoine Guicheteau et Anne-Lise Bugnon-Labaune
}

La réalisation d'un diagnostic archéologique préventif «9, place Occidentale » à Romenay, au mois d'octobre 2017, en préalable à l'aménagement de la terrasse d'un


restaurant, a permis d'enrichir un corpus de données, déjà bien étoffé, concernant le passé de ce village de Bresse, ancienne province localisée entre la Saône et les contreforts du Jura (fig. 1). 
Fig. 1 - Localisation de l'opération
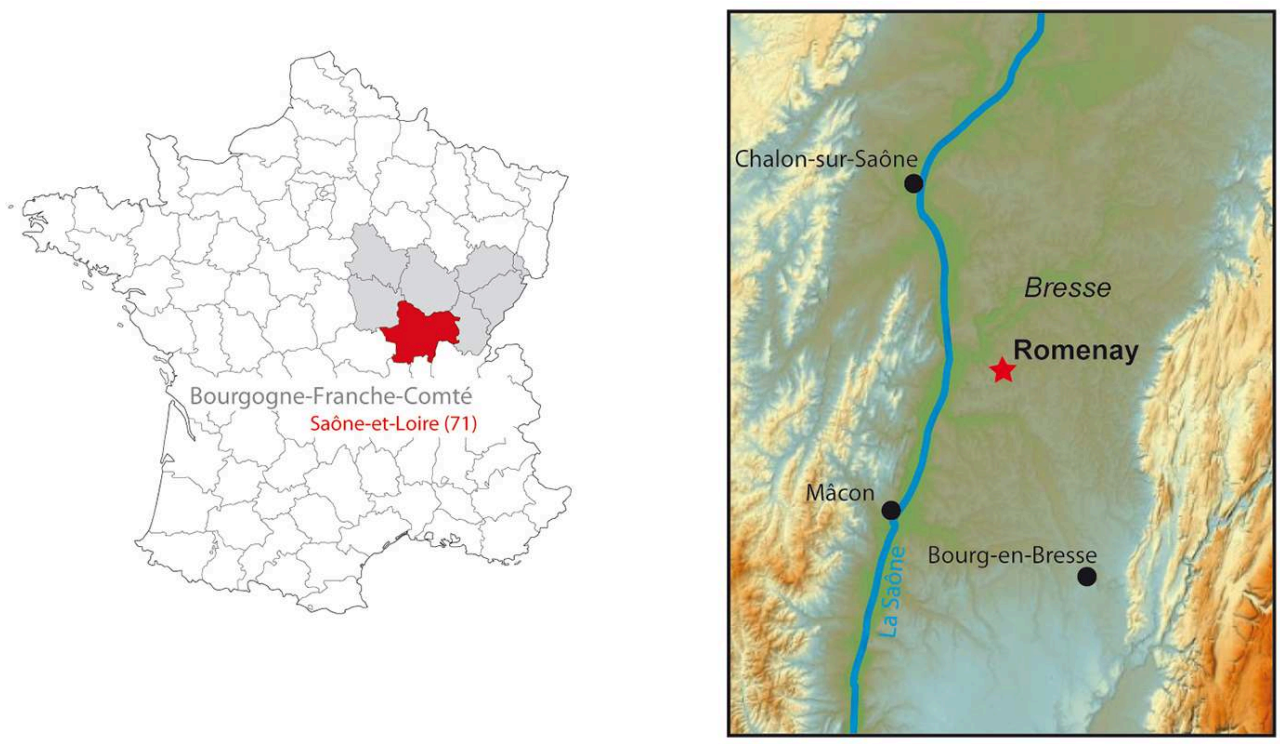

DAO A. Guicheteau; fond de carte géoportail.fr 2017.

2 L'emprise de l'opération - certes limitée à une superficie d'environ $400 \mathrm{~m}^{2}$ - se situe au pied des anciens remparts de briques qui ceinturent le bourg ${ }^{1}$. Les découvertes viennent donc compléter les éléments disponibles. Elles permettent d'esquisser, en corrélation avec les sources écrites et planimétriques, une première synthèse quant à l'évolution de la morphologie de ce village, depuis l'Antiquité jusqu'aux temps contemporains, inscrivant ainsi les éléments de recherche présentés ici dans le mouvement d'étude de la morphogénèse urbaine entamé et défini au tout début des années $1990^{2}$.

\section{Les origines gallo-romaines de Romenay}

Les principaux éléments concernant la période antique proviennent des découvertes réalisées en 1970 lors de la construction d'un lotissement au nord du bourg actuel, au lieu-dit « La Troffetat » (fig. 2). 
Fig. 2 - Plan des vestiges de la période gallo-romaine à Romenay et cliché d'un segment de la voie romaine découverte en 1970
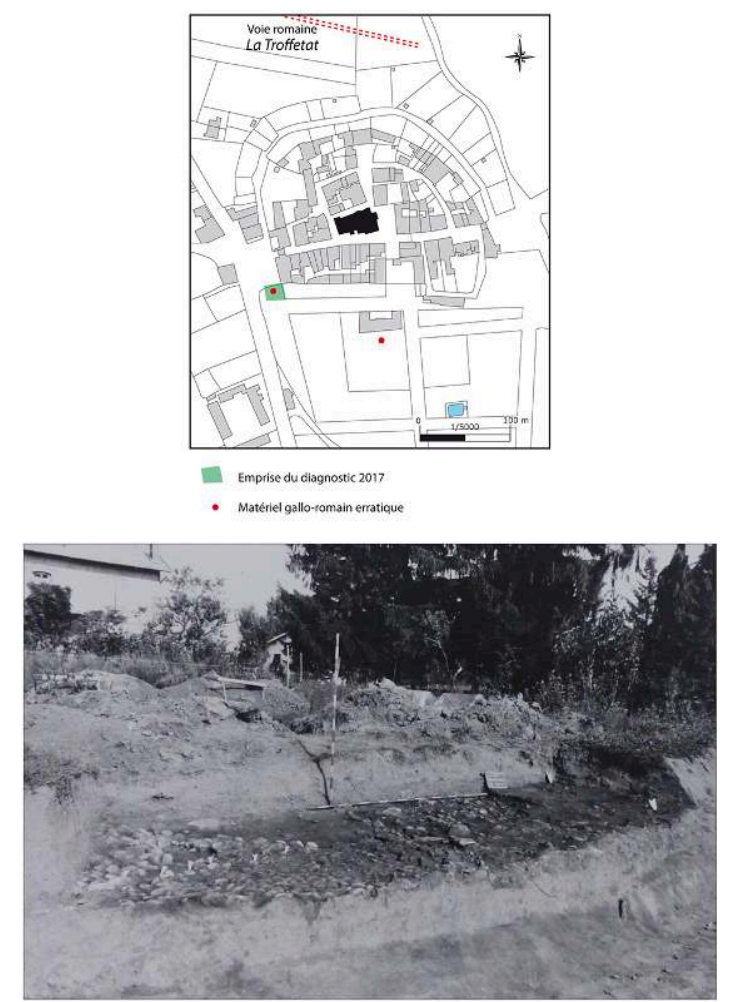

Plan d'après le cadastre de 1833, archives départementales de Saône-et-Loire. DAO A. Guicheteau. Cl. A. Barthélemy, SRA Bourgogne-Franche-Comté.

4 Les vestiges identifiés se composent d'un vaste niveau d'épandage de mobilier disséminé sur l'ensemble de la parcelle, d'une fosse et d'une section de voie empierrée, orientée peu ou prou selon un axe est-ouest ${ }^{3}$. Le matériel recueilli constitue un ensemble attribuable au $\mathrm{II}^{\mathrm{e}}$ siècle de notre ère, comprenant, notamment, des céramiques métallescentes et sigillées (Drag. 35 et 37), une fibule et sept monnaies des Antonins.

Un trésor monétaire a été découvert à la fin $d u \mathrm{XVII}^{\mathrm{e}}$ siècle au lieu-dit « au Clos du Château ", vers le sud du bourg actuel ${ }^{4}$. Il se compose d'une cinquantaine de monnaies, la plupart d'Antonin, de Faustine et de Constantin, indiquant un probable enfouissement durant le premier tiers du IV ${ }^{\mathrm{e}}$ siècle.

Enfin, la présence, manifestement erratique, de deux tessons de céramiques galloromaines, dont un reste de sigillée, dans les niveaux de remblais modernes de la tranchée 1 réalisée lors du diagnostic de 2017, contribue également à illustrer la période antique à Romenay.

\section{La villa Romanaca du haut Moyen Âge}

7 La première mention du village, sous la forme villa Romanaca, remonte à une charte rédigée $\mathrm{au} \mathrm{X}^{\mathrm{e}}$ ou au $\mathrm{XI}^{\mathrm{e}}$ siècle, qui rappelle la donation par Gontran, roi mérovingien de Bourgogne entre 561 et 592/593, de droits sur les serfs de Romenay au chapitre cathédral Saint-Vincent de Mâcon, dépendant de l'évêque de la cité ${ }^{5}$. Ceux-ci doivent alors entretenir les bâtiments de l'évêché, à savoir l'église ainsi que les constructions 
résidentielles et fonctionnelles - [...] et omnes mansiones que ad ipsam curtem aspiciunt, et granicam et stabulum et coquinam. L'origine mérovingienne du bourg est accréditée par l'existence d'une nécropole du haut Moyen Âge autour de l'actuel lieu de culte; les nombreuses réfections ne permettent pas toutefois de faire remonter l'origine de l'édifice au-delà de la période romane (fig. 3).

Fig. 3 - Plan des vestiges de la période alto-médiévale à Romenay et cliché de la porte sud de la nef de l'église paroissiale de Romenay


Plan d'après le cadastre de 1833, archives départementales de Saône-et-Loire. DAO A. Guicheteau. Cl. A. Guicheteau.

8 Le souvenir de la découverte de sarcophages et de sépultures en 1955 lors de la réalisation de travaux d'adduction d'eau au milieu de l'actuelle rue du Colonel Pagand, au pied de l'église, est encore bien vivant chez nombre d'habitants ${ }^{6}$, bien qu'aucun document graphique, dessin ou photographie, ne la documente.

La morphologie actuelle du bourg incite à proposer une première forme d'organisation circulaire de l'habitat. En effet, le parcellaire villageois actuel fossilise encore un arc de cercle au nord-ouest de l'église, ébauche d'un probable enclos d'environ $150 \mathrm{~m}$ de diamètre. À l'instar d'exemples méridionaux, le centre du cercle correspond au milieu du mur pignon de l'église ${ }^{7}$. L'hypothèse d'un enclos ecclésial peut donc être formulée, ce type d'organisation constitue, en effet, une forme originelle tardo-antique du peuplement, comme l'illustrent les nombreux exemples étudiés de longue date dans le Languedoc $^{8}$ ou la fouille d'un tel site à Aoste en Isère ${ }^{9}$. L'implantation de ces établissements au sein des vestiges du réseau antique, notamment en périphérie d'un axe viaire, est également attestée de manière récurrente, à l'exemple du site de Malicorne dans l'Allier ${ }^{10}$. Ce genre d'enclos est fréquemment divisé en différents secteurs - funéraire et religieux, stockage, résidentiel - caractéristiques d'un habitat groupé. Les éléments de datation disponibles sont rares, limités à des sarcophages alto- 
médiévaux ; l'attribution au haut Moyen Âge de la forme parcellaire elliptique fossilisée dans le bâti ne saurait être définitive, même si un texte plus tardif nous permet de situer chronologiquement sa mise en place avant la seconde moitié $\mathrm{du} \mathrm{XII}^{\mathrm{e}}$ siècle.

\section{Bourg fortifié et résidence épiscopale du XII au $\mathrm{XVl}{ }^{\mathrm{e}}$ siècle}

Les sources écrites mentionnent à partir $\mathrm{du}$ XII ${ }^{\mathrm{e}}$ siècle de nombreux travaux de réaménagement et surtout de fortifications du bourg de Romenay par les évêques mâconnais. Un texte indique que Ponce de Thoire, évêque de Mâcon de 1140 à 1161, construit une grange maçonnée à Romenay, ainsi qu'un mur à la place de la haie défensive installée sur un talus ${ }^{11}$. Il s'agit là du témoignage de la construction d'une première enceinte maçonnée en lieu et place d'une enceinte en terre et bois. Un autre texte nous apprend que le successeur de Ponce de Thoire, Étienne de Bâgé (1164-1184), ferme Romenay, en poursuivant vraisemblablement la construction de l'enceinte en dur ; il fait également construire un nouvel édifice entre la chapelle et la grange bâtie par son prédécesseur ${ }^{12}$. Par ailleurs, le texte renseigne l'existence d'une motte aujourd'hui disparue. Il est possible de la localiser au sud-ouest du bourg, d'après la description que fait de Romenay Gabriel de Chavanel en $1697^{13}$ et la représentation graphique en vue cavalière du village réalisée au début du xvIII ${ }^{e}$ siècle (fig. 4).

Fig. 4 - Vue cavalière de Romenay vers 1730

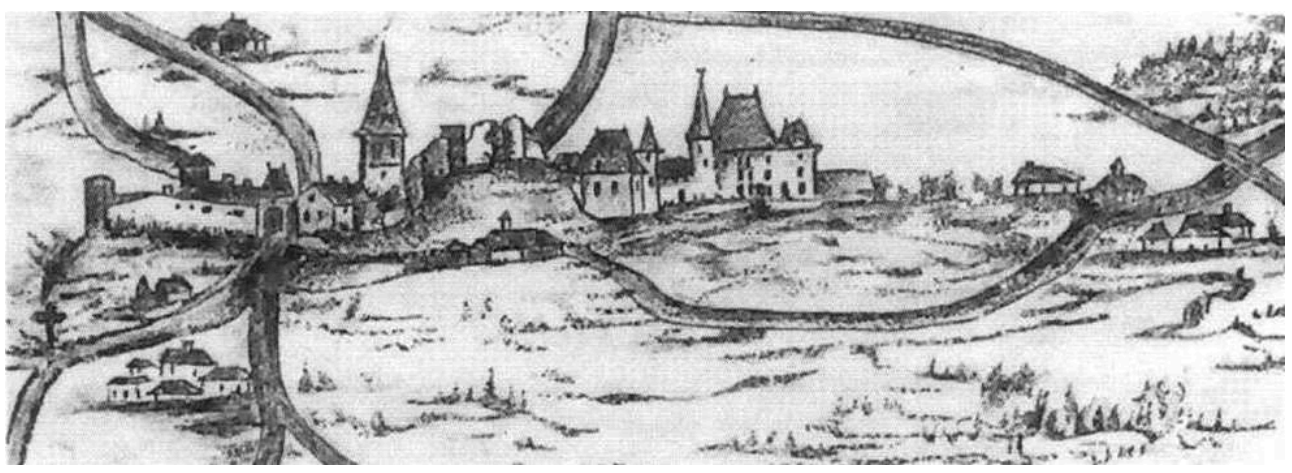

Collection particulière de la famille De La Chaise.

De nouveaux travaux, concernant les fortifications et les bâtiments résidentiels et fonctionnels d'un véritable château en lieu et place de la motte, sont également attestés durant le séjour de Renaud de Vergy (1185-1197) sur le siège épiscopal de Mâcon ${ }^{14}$.

De ce rempart subsistent aujourd'hui des segments de courtines, deux portes et deux tours conservés dans le bâti actuel du bourg (fig. 5). 
Fig. 5 - Plan des vestiges de la période médiévale à Romenay et clichés des éléments du rempart actuellement conservés

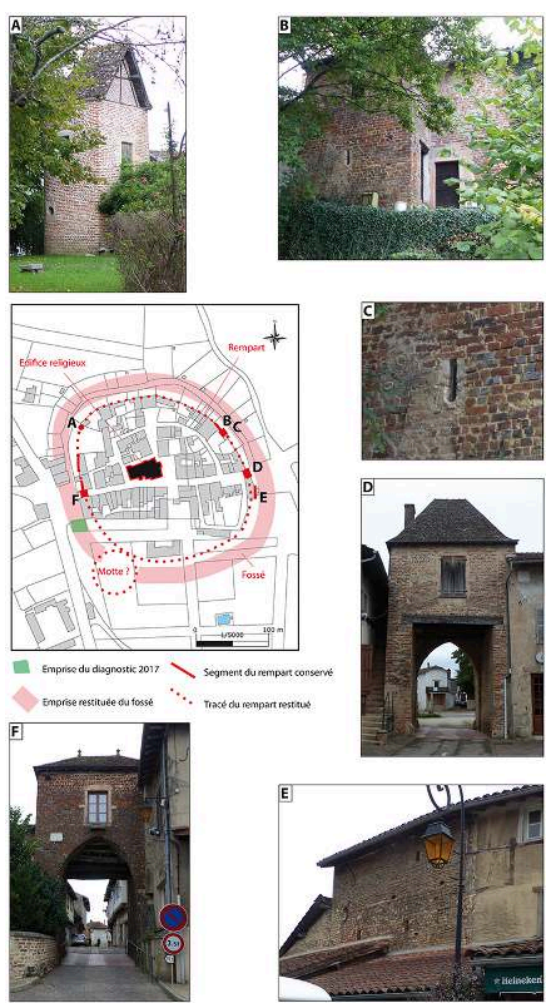

A : tour circulaire, B : tour carrée, C : ouverture de tir au sein de la tour carrée, D : porte orientale, E : segment de courtine, $\mathrm{F}$ : porte occidentale.

Plan d'après le cadastre de 1833, archives départementales de Saône-et-Loire. DAO A. Guicheteau. Cl. A. Guicheteau.

Ces éléments matérialisent une seconde ceinture circulaire autour de la première, très probablement postérieure à la première ellipse. L'originalité de ces fortifications consiste en leur réalisation entièrement en briques et mortier. En effet, faute de ressources en pierre en Bresse, l'argile a longtemps constitué avec le bois le seul matériau utilisable pour la construction. Cette caractéristique de l'architecture bressane se retrouve par exemple dans les remparts de Louhans ${ }^{15}$. Dans son état actuel, le rempart de briques daterait du XIV ${ }^{e}$ siècle, mais l'absence de texte indiquant une nouvelle construction, a contrario des nombreuses mentions du XII ${ }^{\mathrm{e}}$ siècle, suggère une chronologie plus ancienne ne serait-ce que pour le tracé originel de la fortification. En outre, les maçonneries actuelles portent les stigmates de l'évolution de l'art de la guerre, ce qu'illustre le percement d'ouvertures de tir, comme celle repérée dans un segment de courtine à l'est du bourg.

14 Le diagnostic de 2017 a permis de préciser le tracé du fossé sis en avant des remparts. Il se développe selon un axe NNO-SSE, différent de celui du bâti actuel, sur une largeur d'une dizaine de mètres au minimum, seule son extrémité orientale ayant pu être appréhendée (fig. 6). 
Fig. 6 - Plan du diagnostic de 2017 « 9, place occidentale », coupes du fossé d'enceinte, relevé des bords de marmite - dont un à anse coudée (3) - à pâte grise des $\mathrm{XV} l^{\mathrm{e}}-\mathrm{XV} \|^{\mathrm{e}}$ siècles recueillis dans le remplissage du fossé dans la tranchée 3 , et cliché vers le sud du début du creusement du fossé d'enceinte dans la tranchée 1


Coupes du fossé d'enceinte : DAO A. Guicheteau. Relevé des bords de marmite : dessin et DAO A.-L. Bugnon-Labaune. Cl. A. Guicheteau.

Le fond du creusement n'a pu être atteint, aussi une profondeur de 1,90 m apparait bien là encore comme un minimum. L'ouvrage apparait donc massif, avec un rôle militaire réel. Il s'agit, en effet, de se prémunir des conséquences des troubles qui impactent la Bresse à partir de la fin du XIII ${ }^{\mathrm{e}}$ siècle, avec les guerres entre la Savoie et le Dauphiné, puis la guerre de Cent Ans, les guerres de Religion du Xvi ${ }^{\mathrm{e}}$ siècle, ainsi que les conflits avec les Habsbourgeois, implantés en Franche-Comté, et les Savoyards. Dès lors, Romenay est fréquemment attaqué, pillé, et les destructions sont sans doute récurrentes sans être pour autant nécessairement importantes, mais suffisantes pour cantonner le développement du bourg derrière ses remparts.

\section{Nouveaux développements aux périodes moderne et contemporaine}

La fin de la période moderne voit, en effet, Romenay connaître un nouveau programme de constructions. L'ancien château est abattu, la motte arasée et les fossés comblés. Le matériel recueilli au sein du comblement du fossé indique une fermeture progressive de la structure durant les temps modernes. Les restes céramiques recueillis consistent essentiellement en des productions sevrotines, en l'occurrence des bords de marmites, dont un à anse coudée, attribuables à une période comprise entre la seconde moitié du $\mathrm{XVI}^{\mathrm{e}}$ siècle et le courant du XVII ${ }^{\mathrm{e}}$ siècle ${ }^{16}$. Par ailleurs, la présence de scories de fer -34 
restes totalisant une masse de $2806 \mathrm{~g}$ issus d'un ramassage non exhaustif - découvertes dans un colmatage du fossé, situé sur le flanc occidental du creusement, témoigne du déroulement d'activités métallurgiques. La forte fragmentation des pièces, leur caractère aéré et leur forte réaction à l'aimantation semblent permettre de les considérer comme des déchets de forges. Ces matériaux illustrent de nouveau le rejet des artisanats polluants hors des murs et des zones d'habitations ${ }^{17}$.

Une nouvelle résidence épiscopale s'installe au sud du bourg, sans doute en lieu et place de l'ancien château. Le bâti de la partie méridionale du village est alors reconstruit selon une nouvelle orientation inscrite en deux axes, l'un nord-sud et l'autre est-ouest, selon le modèle de la demeure d'agrément et des jardins à la française qui sont alors réalisés. L'hypothèse d'un ensemble architectural clos peut être avancée du fait de la découverte d'un segment de mur en briques, prises dans un mortier sableux beige localisé dans le prolongement de la limite sud du bâti du bourg, à l'endroit d'une limite parcellaire figurée sur le cadastre de 1833 et disparue aujourd'hui. Toujours dans un même souci d'ouverture de l'espace et de dégagement des perspectives, une place est créée face à la résidence de l'évêque (fig. 7).

Fig. 7 - Plan des vestiges de la période moderne à Romenay, cliché vers l'ouest du segment de mur maçonné en briques et des fondations d'un plot en briques découverts dans la tranchée 2 du diagnostic de 2017 (A) et cliché de la résidence épiscopale du XVIII ${ }^{e}$ siècle, actuelle mairie, vers le sud-est (B)
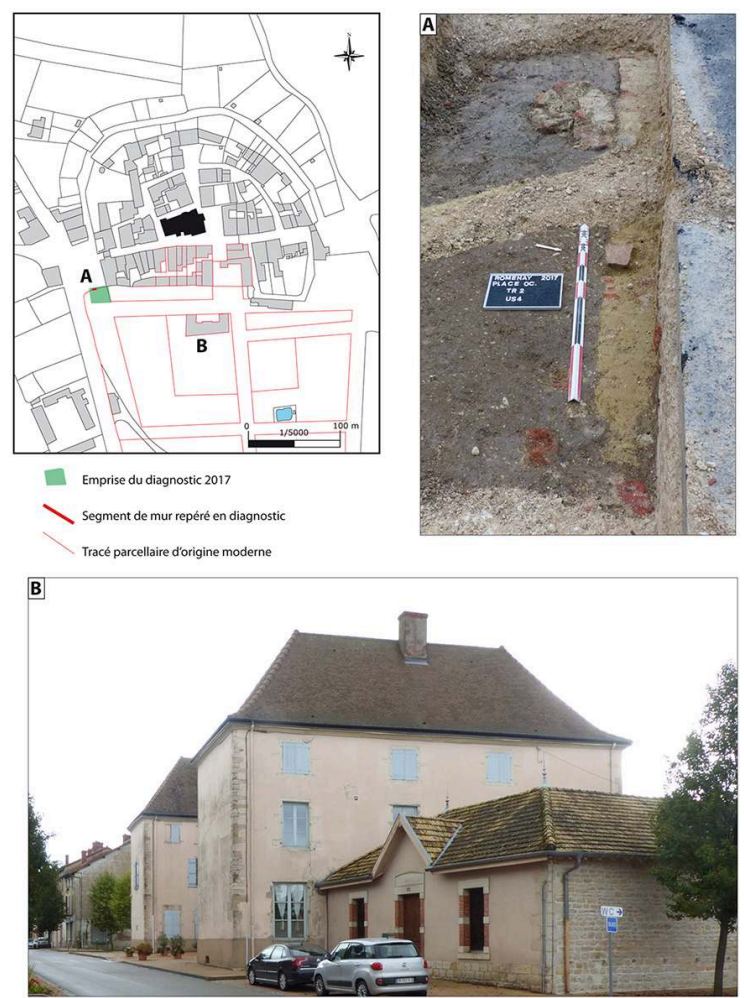

Plan d'après le cadastre de 1833, archives départementales de Saône-et-Loire. DAO A. Guicheteau. Cl. A. Guicheteau.

18 Au cours de la période contemporaine, les formes du bâti du bourg n'évoluent guère, mais les espaces changent de fonction. Les biens de l'évêché sont sécularisés: la résidence proprement dite devient la mairie, tandis que les communs sont transformés en école. Les jardins à la française du parc d'agrément sont pour partie lotis, pour 
partie transformés en place de marché pour les foires à bestiaux de la Bresse. Les données du diagnostic illustrent, en effet, un remblaiement général des terrains au $\mathrm{XIX}^{\mathrm{e}}$ siècle, comme en témoignent les restes de faïence fine récupérés ${ }^{18}$ et la destruction du probable mur de clôture. La présence de la carcasse d'un jeune équidé dans les niveaux de remblais du XIX ${ }^{e}$ siècle, datation corrélée par la robustesse de l'animal ${ }^{19}$, accrédite le déroulement de foires aux bestiaux dans ce secteur du bourg. En témoigne également la construction en 1900 d'une bascule servant à la pesée des bestiaux sur la place de foire, renommée «du Monuments aux morts » depuis la fin de la première guerre mondiale, quelques mètres au sud-est.

\section{L'évolution des formes bâties d'un habitat groupé en pays de plaine, entre ruptures et héritages}

19 L'analyse des formes parcellaires, corrélée aux données archéologiques et aux sources textuelles, permet de percevoir les différents mouvements d'évolution du bourg de Romenay, depuis la période gallo-romaine jusqu'à nos jours. En l'état, bien qu'il demeure délicat d'appréhender les origines antiques du bourg, l'hypothèse d'une continuité du peuplement jusque dans le haut Moyen Âge semble à privilégier. La donation du roi Gontran à l'évêché de Mâcon marque cependant une étape probablement décisive dans la genèse de l'habitat, avec l'implantation d'un pôle religieux et funéraire. Les limites de cet espace au statut indéterminé, mais peut-être ecclésial, s'inscrivent sans doute dans le paysage sous la forme d'un premier enclos circulaire. Dans le courant du Moyen Âge, entre la fin du VI et le milieu du XII siècle, l'espace s'avère trop étroit, et le bourg s'étend sous la forme d'un anneau ceinturant l'enclos initial. Ces nouvelles limites sont probablement marquées par un système de fortifications en terre et bois évoqué par les textes, que vient compléter une motte castrale, avec son cortège de bâtiments résidentiels et économiques. Romenay est alors une résidence des évêques de Mâcon. Dans un souci d'efficacité militaire, et sans doute aussi de prestige, un rempart de briques est construit par la suite, accompagné d'un imposant fossé. Son origine remonte peut-être à la fin du XII siècle, mais l'ensemble ne cesse d'évoluer au fil du temps. Les exigences de confort formulées par les élites de la période moderne se traduisent à Romenay par la réalisation d'un nouveau programme architectural d'envergure au début du $\mathrm{xVIII}^{\mathrm{e}}$ siècle. Les fortifications sont donc largement démantelées et un nouvel ensemble résidentiel est construit au sud du bourg. Avec le XIX ${ }^{e}$ siècle et son vaste mouvement de sécularisation, ces espaces, relevant de l'évêque de Mâcon, deviennent des espaces publics et connaissent alors leur ultime évolution.

Reçu : 27 février 2018 - Accepté : 25 août 2018 


\section{NOTES}

1. A. Guicheteau, avec A.-L. Bugnon-Labaune, Romenay, Saône-et-Loire, " 9, place Occidentale ", regard sur la morphogenèse de Romenay, de l'Antiquité à nos jours, rapport de diagnostic, DRAC/SRA Bourgogne-Franche-Comté, Dijon, 2017.

2. G. FABRE, M. BOURIN, J. CAILLE et A. DEBORD (dir.), Morphogenèse du village médiéval: IX ${ }^{e}-X I I^{e}$ siècles, Montpellier, 1996 (Cahiers du Patrimoine, 46) ; B. GAUTHIEZ, E. ZADORA-RIO et H. GALINIÉ (dir.), Village et ville au Moyen Âge, les dynamiques morphologiques, Tours, 2003.

3. A. BARTHÉLEMY, «Découvertes archéologiques en Tournugeois », Bulletin du Groupe de recherche archéologique de Tournus, 7 (1978).

4. A. Rebourg (dir.), Carte archéologique de la Gaule, T. 71/4 (Saône-et-Loire), Paris, 1994, p. 455-459.

5. M.-C. RAGUT, Cartulaire de Saint-Vincent de Mâcon connu sous le nom de livre enchaîné, Mâcon, 1864, charte CCCCXCIII, p. 286.

6. Témoignage oral de M. Ballot, antiquaire à Romenay et membre de l'association Les amis du vieux Romenay.

7. Ainsi l'exemple de Bram dans l'Aude, p. 133-134, cf. A. QUERRIEN, « Les formes circulaires de l'espace bâti et agricole au Moyen Âge: tracé, mesure et partage", Archéologie médiévale, 38 (2008), p. 123-158, en particulier p. 133-134.

8. Voir quelques exemples présentés récemment dans A.CATAFAU et O. PASSARIUS, " "Village ecclésial" et cellera en Languedoc-Roussillon : questions en débat et éclairages archéologiques ", in C. TREFFoRT (dir.), Le cimetière au village dans l'Europe médiévale et moderne, Toulouse, 2015, p. 107-124.

9. M.-J. ANCEL, «Un enclos ecclésial du haut Moyen Âge à Aoste "Les Communaux - ZAC PIDA" (Isère) », Bulletin de liaison de l'AFAM, 41 (2017), p. 98-101.

10. Cf. A. QUERRIEN, « Les formes circulaires... », op. cit., p. 131.

11. P. Gras et J. LAURENT, Obituaires de la province de Lyon, T. 2, Paris, 1965, p. 406C. Par ailleurs, un important corpus de textes documentant l'histoire médiévale et moderne de Romenay est disponible sur la base de données en ligne «L'habitat médiéval fortifié en Bourgogne ", réalisée par le Centre de castellologie de Bourgogne (CeCaB) dirigé par Hervé Mouillebouche (http:// 193.52.240.114/gorria/QooQ4D/chateaux.html?tous).

12. P. Gras et J. Laurent, Obituaires..., ibid., p. 415DE.

13. G. ChAVANEL, Histoire de Romenay, Mâcon, 1698 (rééd. 1986).

14. P. Gras et J. LAURENT, Obituaires..., op. cit., p. 393C.

15. A. BLETON-RUGET et C. PACZYNSKI, «Les fortifications de Louhans : première approche », in $B$. Colas et H. Mouillebouche (dir.), Chastels et maisons fortes, Chagny, 2014, p. 259-271.

16. A. HorrY, Poteries du quotidien en Rhône-Alpes, XVI ${ }^{e}, X V I I^{e}, X_{I I I}{ }^{e}$ siècles. Un panorama des techniques des formes et des décors, Lyon, 2015 (DARA, 43), p. 28.

17. Y. HeNIGFELD et A. MASQUILIER (dir.), Archéologie des enceintes urbaines et de leurs abords en Lorraine et en Alsace (XII ${ }^{e}-\mathrm{XV}^{e}$ siècle), Dijon, 2008 (RAE, $26^{\mathrm{e}}$ supplément), p. 445.

18. A. Horry, Poteries du quotidien..., op. cit., p. 108.

19. Nous remercions David Cambou, archéozoologue à l'Inrap, pour ses observations. 
INDEX

Mots-clés : Antiquité, haut Moyen Âge, Moyen Âge central, temps modernes, période contemporaine, église paroissiale, village, enceinte, fossé, Bresse

\section{AUTEURS}

ANTOINE GUICHETEAU

Responsable d'opération, Inrap, UMR 6298-ArTeHiS

\section{ANNE-LISE BUGNON-LABAUNE}

Céramologue, Inrap, UMR 6298-ArTeHiS 